\title{
Abbreviation of Chinese Botanical Garden Names
}

Name

Anhui Biological Institute Botanical Garden

Anji Bamboo Garden

Anshan 219 Botanical Garden

Baoding Botanical Garden

Baoji Botanical Garden

Baotou Landscape Institute Arboretum

Bayi Agricultural University Wild Economic Botanical Garden

Beijing Botanical Garden, CAS

Beijing Education Botanical Garden

Beijing Medicinal Botanical Garden

Beijing Municipal Botanical Garden

Central South University of Forestry and Technology Botanical Garden

Changbaishan Botanical Garden

Changchun Forestry Botanical Garden

Changchun Zoo and Botanical Garden

Chaozhou Botanical Garden

Chengdu Botanical Garden

Chifeng Arboretum

Chongqing Botanical Garden

Chongqing Flower Garden

Chongqing Medicinal Botanical Garden

Chongqing Nanshan Botanical Garden

Chongqing Three Gorges Precious \& Rare Botanical Garden

Dalian Botanical Garden

Dalian Yinggeshi Botanical Garden

Datong Botanical Garden

Daxing'anling Frigid-temperate Forestry Botanical Garden

Dengkou Psammophyte Botanical Garden

Dezhou Botanical Garden

Dinghushan Mountain Arboretum

Dongfeng Forestry Botanical Garden

Dongguan Botanical Garden

Donghu Moshan Landscape Botanical Garden

\author{
Abbreviation \\ Anhui I BG \\ Anji B BG \\ Anshan 219 BG \\ Baoding BG \\ Baoji BG \\ Baotou L Arb \\ Bayi WE BG \\ Beijing (I) BG \\ Beijing Edu BG \\ Beijing M BG \\ Beijing (M) BG \\ Central South F BG \\ Changbaishan BG \\ Changchun F BG \\ Changchun Z BG \\ Chaozhou BG \\ Chengdu BG \\ Chifeng Arb \\ Chongqing BG \\ Chongqing F G \\ Chongqing $\mathrm{M} B \mathrm{BG}$ \\ Nanshan BG \\ Three Gorges PR BG \\ Dalian BG \\ Yinggeshi BG \\ Datong BG \\ Daxing'anling FF BG \\ Dengkou Ps BG \\ Dezhou BG \\ Dinghushan Arb \\ Dongfeng F BG \\ Dongguan BG \\ Donghu ML BG
}


Dongtai Caost Economic Botanical Garden

Dongtai C BG

Dujuancun Botanical Garden

Dujuancun BG

Fushan Botanical Garden

Fushan BG

Fuzhou Arboretum

Fuzhou Arb

Guangxi Liuzhou Karst Botanical Garden

Liuzhou K BG

Guangxi Medicinal Botanical Garden

Guangxi M BG

Guangxi Shishan Arboretum

Shishan Arb

Guilin Botanical Garden

Guilin BG

Guizhou Botanical Garden

Guizhou BG

Guizhou Forestry Academy Arboretum

Guizhou F Arb

Guizhou Kaili Arboretum

Kaili Arb

Guizhou Medicinal Botanical Garden

Guizhou M BG

Guizhou Xiaolongli Subtropical Alpine Botanical Garden

Xiaolongli BG

Guizhou Zunyi Arboretum

Zunyi Arb

Hainan Forestry Institute Arboretum

Hainan Arb

Hainan Tropical Economic Botanical Garden

Hainan BG

Hangzhou Botanical Garden

Hangzhou BG

Hangzhou Medicinal Botanical Garden

Hangzhou M BG

Hefei Botanical Garden

Hefei BG

Heilongjiang Forestry Botanical Garden

Heilongjiang F BG

Heilongjiang Shihe Arboretum

Shihe Arb

Hengchun Tropical Botanical Garden

Hengchun T BG

Hong Kong Kadoorie Farm \& Botanic Garden

Kadoorie F BG

Hong Kong Ocean Park

HK Ocean P

Hong Kong Zoological \& Botanical Park

Hongkong Z BP

Huanghe Tourism District Botanical Garden

Huanghe T BG

Huangshan Arboretum

Huangshan Arb

Huaxi Subalpine Botanical Garden

Huaxi Sa BG

Hohhot Arboretum

Hohhot Arb

Hohhot Botanical Garden

Hohhot BG

Hunan Forestry Botanical Garden

Hunan F BG

Hunan Forestry Institute Nanling Botanical Garden

Nanling BG

Hunjiang Arboretum

Hunjiang Arb

Jianfengling Tropical Botanical Garden

Jianfengling T BG

Jiangxi Forestry Academy Nanchang Arboretum

Nanchang Arb

Jiayi Arboretum

Jiayi Arb

Jigongshan Mountains Botanical Garden

Jigongshan M BG

Jinan Botanical Garden

Jinan BG

Jiuhuashan Botanical Garden

Jiuhuashan BG

Kaiyuan Tropical Botanical Garden 
Kowloon City-wall Gate Arboretum

Kunming Botanical Garden

Kunming Landscape Botanical Garden

Laizhou Precious \& Rare Plant Arboretum

Lanzhou Arboretum

Lanzhou Xerophyte Botanical Garden

Lijiang Botanical Garden

Luoyang Botanical Garden

Luoyang Sui-Tang Ruins Botanical Garden

Lushan Botanical Garden

Maijishan Arboretum

Minqin Psammophyte Botanical Garden

Nanjing Botanical Garden Mem. Sun Yat Sen

Nanjing Forestry University Arboretum

Nanning Arboretum

Nanning People's Park Shaded Foliage Botanical Garden

Nanyue Arboretum

Qingdao Botanical Garden

Qinling Botanical Garden

Rudong Salt-tolerant Plant Botanical Garden

Second Military Medical University Botanical Garden

Shandong Forestry School Arboretum

Shandong Linyi Zoo \& Botanical Garden

Shanghai Botanical Garden

Shanghai Chenshan Botanical Garden

Shapotou Botanical Garden

Shenyang Applied Ecology Botanical Garden, CAS

Shenyang Botanical Garden

Shenyang Landscape Institute Arboretum

Shenyang Pharmaceutical University Botanical Garden

Shenzhen Fair Lake Botanical Garden

Shenzhen Longgang Botanical Garden

Shijiazhuang Botanical Garden

Shuangxi Arboretum

South China Botanical Garden

Southern Subtropical Botanical Garden

Subtropical Forestry Institute Arboretum, CFA

Taipei Botanical Garden

Tianjin Tropical Ornamental Garden

Turpan Desert Botanical Garden
Kowloon CG Arb

Kunming BG

Kunming L BG

Laizhou PR Arb

Lanzhou Arb

Lanzhou Xr BG

Lijiang BG

Luoyang BG

Luoyang SR BG

Lushan BG

Maijishan Arb

Minqin Ps BG

Nanjing BG

Nanjing F Arb

Nanning Arb

Nanning SF BG

Nanyue Arb

Qingdao BG

Qinling BG

Rudong St BG

Sec Min M BG

Shandong F Arb

Linyi Z BG

Shanghai BG

Sh Chenshan BG

Shapotou BG

Shenyang Eco BG

Shenyang BG

Shenyang L Arb

Shenyang Ph BG

Shenzhen BG

Longgang BG

Shijiazhuang BG

Shuangxi Arb

South China BG

S Subtropical BG

Subtropical F Arb

Taipei BG

Tianjin T OG

Turpan D BG 


\section{xviii}

Urumqi Botanical Garden

Weixi Yongchun Snow Mountain Botanical Garden

Wenzhou Botanical Garden

Wuhan Botanical Garden

Wuhan University Arboretum

Wuhan University of Hydraulic \& Electrical Engineering Arboretum

Wutaishan Arboretum

Wuxi Taihu Ornamental Botanical Garden

Xiamen Botanical Garden

Xiamen Overseas' Subtropical Plant Introduction Garden

Xian Botanical Garden

Xiaoxing'anling Forestry Arboretum

Xiji Botanical Garden

Xinglong Tropical Botanical Garden

Xinglong Tropical Flower Garden

Xining Botanical Garden

Xiongyue Arboretum

Xishaungbanna Medicinal Botanical Garden

Xishuangbanna Tropical Botanical Garden

Xishuangbanna Tropical Flower Garden

Yan'an Arboretum

Yanbei Semiarid Botanical Garden

Yanbian Maoershan Botanical Garden

Yanchi Sandy \& Arid Areas Arboretum

Yichang Three Gorges Botanical Garden

Yili Prefecture Forestry Institute Arboretum

Yinchuan Botanical Garden

Yingkou Botanical Garden

Yixing Precious \& Rare Botanical Garden

Yulin Heilongtan Mountain Arboretum

Yulin Sandy Land Botanical Garden

Yulin Woyunshan Private Botanical Garden

Zhangjiajie Arboretum

Zhejiang Agricultural University Botanical Garden, Huajiachi

Zhejiang Bamboo Botanical Garden

Zhejiang Forestry College Botanical Garden

Zhengzhou Botanical Garden

Zhongdian Alpine Botanical Garden

Zhoushan Island Plant Introduction \& Acclimatization Arboretum
Urumqi BG

Weixi SN BG

Wenzhou BG

Wuhan BG

Wuhan Arb

Wuhan HE Arb

Wutaishan Arb

Wuxi Taihu O BG

Xiamen BG

Xiamen OS BG

Xian BG

Xiaoxing'anling F Arb

Xiji BG

Xinglong T BG

Xinglong TF G

Xining BG

Xiongyue Arb

Xishaungbanna M BG

Xishuangbanna T BG

Xishuangbanna TF G

Yan'an Arb

Yanbei Sa BG

Maoershan BG

Yanchi SA Arb

Yichang TG BG

Yili F Arb

Yinchuan BG

Yingkou BG

Yixing PR BG

Yulin Hlt Arb

Yulin BG

Woyunshan P BG

Zhangjiajie Arb

Huajiachi BG

Zhejiang B BG

Zhejiang F BG

Zhengzhou BG

Zhongdian A BG

Zhoushan IP Arb 